Special issue of the International Conference on Computational and Experimental Science and Engineering (ICCESEN 2014)

\title{
Gluon Energy at Next-to-Leading Order in Hot QCD Using the Real-Time Formalism
}

\author{
K. Benchallat ${ }^{a, *}$, A. Abada ${ }^{a, b}$ And K. Bouakaz ${ }^{a}$ \\ ${ }^{a}$ Physics Department, Laboratoire de Physique des Particules et de Physique Statistique, Ecole Normale Supérieure, \\ PB 92, Alger 16050, Algeria \\ ${ }^{b}$ Department of Physics, United Arab Emirates University, PB 17551, Al Ain, United Arab Emirates

\begin{abstract}
We give the analytic expression of the next-to-leading order contribution to the longitudinal gluons energy in high-temperature quantum chromodynamic using the real-time formalism of finite-temperature quantum field theory. We also give the expressions of the effective propagators and vertex functions.
\end{abstract}

DOI: 10.12693/APhysPolA.128.B-290

PACS: 11.10.Wx, 12.38.- - 1 , 12.38.Mh

\section{Introduction}

The problem of gauge dependence of the physical quantities in high-temperature perturbation theory of quantum chromodynamics (QCD) was resolved by Braaten and Pisarski in [1]. They showed that in order to calculate consistently at high temperature, we have to use an effective perturbation that sums the so-called hard thermal loops (HTL) into dressed propagators and vertices $[1,2]$. The first next-to-leading order physical quantity that has been determined in this framework is the damping rate of the non-moving transverse gluon [3]. Indeed, it was shown to be finite, positive and gaugeindependent.

However, in the imaginary-time formalism, infrared difficulties occur in next-to-leading order HTL-summed perturbation. This was first indicated in [4], where the direct and explicit attempt to calculate the damping rate for non-moving longitudinal gluons showed that it is infrared divergent. The infrared sensitivity occurs in other quantities too $[5,6]$.

\section{Model and results}

The present work gives the analytic expression of the longitudinal-gluon energy at next-to-leading order in HTL-summed perturbation of massless QCD at high temperature. The real-time formalism $[2,7-9]$ is used instead of the imaginary-time one. It also gives expressions for the vertex functions using the Feynman parameterization.

In the physical representation, the retarded/advanced (ra|ar) effective gluon propagators are given by these expressions:

$$
{ }^{*} \Delta_{\mathrm{ra} \mid \mathrm{ar}}^{l}(P, Q)=\frac{1}{p^{2}-\delta \Pi_{\mathrm{ra} \mid \mathrm{ar}}^{l}(P, Q)},
$$

*corresponding author; e-mail: benchallal.k@gmail.com

$$
{ }^{*} \Delta_{\mathrm{ra} \mid \mathrm{ar}}^{t}(P, Q)=\frac{1}{P^{2} \pm \mathrm{i} \varepsilon-\delta \Pi_{\mathrm{ra} \mid \mathrm{ar}}^{t}(P, Q)} .
$$

The quantities $\delta \Pi_{\mathrm{ra} \mid \mathrm{ar}}(P, Q)$ are the HTL contributions to the retarded/advanced gluon self-energy.

The poles of $* \Delta^{l(t)}$ determine the longitudinal (transverse) gluon dispersion relations to lowest order $g T$. The next-to-leading order dispersion relation of the longitudinal gluons is given by the following relation:

$$
p^{2}-\delta \Pi_{l}\left(\Omega_{l}, p\right)-{ }^{*} \Pi_{l}\left(\Omega_{l}, p\right)=0 .
$$

In this expression, the quantity ${ }^{*} \Pi_{l}\left(\Omega_{l}, p\right)$ is the nextto-leading order longitudinal gluon self-energy. From this dispersion relation, the next-to-leading-order energy of the slow-moving longitudinal gluons can be cast as follows:

$$
\operatorname{Re} \Omega_{l}(p)=w_{l}(p)-\frac{\operatorname{Re}^{*} \Pi_{\mathrm{ra} \mid \mathrm{ar}}^{l}(P)}{\frac{\partial}{\partial w} \delta \Pi_{\mathrm{ra} \mid \mathrm{ar}}^{l}(w, P)} .
$$

The denominator in this expression is easy to obtain whereas the numerator requires a derivation of the real part of the effective self-energy.

In the strict Coulomb gauge, the compact expression of the retarded component of the next-to-leading order longitudinal gluon self-energy is given by:

$$
\begin{aligned}
& { }^{*} \Pi_{\mathrm{ra}}^{l}(P, Q)= \\
& \quad \frac{g^{2} N_{c}}{2} \int \frac{\mathrm{d}^{4} k}{(2 \Pi)^{4}}\left[{ }^{*} \Gamma_{\mathrm{ra} \mid \mathrm{ar}}^{00 \lambda \sigma}(P,-P, K,-K)^{*} \Delta_{\mathrm{ar}}^{\lambda \sigma}(K)\right. \\
& +{ }^{*} \Gamma_{\mathrm{rara}}^{00 \lambda \sigma}(P,-P, K,-K)^{*} \Delta_{\mathrm{ra}}^{\lambda \sigma}(K) \\
& +{ }^{*} \Gamma_{\mathrm{rarr}}^{00 \lambda \sigma}(P,-P, K,-K)^{*} \Delta_{\mathrm{rr}}^{\lambda \sigma}(K) \\
& +{ }^{*} \Gamma_{\mathrm{rar}}^{0 \lambda \sigma}(P,-K,-Q)^{*} \Delta_{\mathrm{ar}}^{\lambda \lambda^{\prime}}(K) \\
& \quad{ }^{*} \Gamma_{\mathrm{rar}}^{\lambda^{\prime} 0 \sigma^{\prime}}(-K, P,-Q)^{*} \Delta_{\mathrm{rr}}^{\sigma \sigma^{\prime}}(Q) \\
& +{ }^{*} \Gamma_{\mathrm{rra}}^{0 \lambda \sigma}(P,-K,-Q)^{*} \Delta_{\mathrm{rr}}^{\lambda \lambda^{\prime}}(K) \\
& \quad{ }^{*} \Gamma_{\mathrm{rar}}^{\lambda^{\prime} 0 \sigma^{\prime}}(-K, P,-Q)^{*} \Delta_{\mathrm{ar}}^{\sigma \sigma^{\prime}}(Q) \\
& +{ }^{*} \Gamma_{\mathrm{raa}}^{0 \lambda \sigma}(P,-K,-Q)^{*} \Delta_{\mathrm{ar}}^{\lambda \lambda^{\prime}}(K)
\end{aligned}
$$




$$
\begin{aligned}
& \times^{*} \Gamma_{\mathrm{rar}}^{\lambda^{\prime} 0 \sigma^{\prime}}(-K, P,-Q)^{*} \Delta_{\mathrm{ar}}^{\sigma \sigma^{\prime}}(Q) \\
& { }^{*} \Gamma_{\mathrm{rar}}^{0 \lambda \sigma}(P,-K,-Q)^{*} \Delta_{\mathrm{ar}}^{\lambda \lambda^{\prime}}(K) \\
& \times^{*} \Gamma_{\mathrm{raa}}^{\lambda^{\prime} 0 \sigma^{\prime}}(-K, P,-Q)^{*} \Delta_{\mathrm{ra}}^{\sigma \sigma^{\prime}}(Q) \\
& { }^{*} \Gamma_{\mathrm{rra}}^{0 \lambda \sigma}(P,-K,-Q)^{*} \Delta_{\mathrm{ra}}^{\lambda \lambda^{\prime}}(K) \\
& \left.\times{ }^{*} \Gamma_{\mathrm{aar}}^{\lambda^{\prime} 0 \sigma^{\prime}}(-K, P,-Q)^{*} \Delta_{\mathrm{ar}}^{\sigma \sigma^{\prime}}(Q)\right] .
\end{aligned}
$$

Each contribution of the four-gluon vertex functions has the following explicit expression:

$$
\begin{aligned}
& { }^{*} \Gamma^{00 \lambda \sigma \prime}(P,-P, K,-K)^{*} \Delta^{\lambda \sigma}(K) \equiv \\
& \quad{ }^{*} \Gamma^{0000 *} \Delta^{l}+{ }^{*} \Gamma^{00 i j}\left(-g^{i j}+k^{i} k^{j}\right)^{*} \Delta^{t}
\end{aligned}
$$

and each contribution of the three-gluon vertex functions has this expression:

$$
\begin{aligned}
{ }^{*} & \Gamma^{\sigma 0 \lambda}(-Q, P,-K)^{*} \Delta^{\lambda \lambda^{\prime}}(K) \\
& \times{ }^{*} \Gamma^{\lambda^{\prime} 0 \sigma^{\prime}}(-K, P,-Q)^{*} \Delta^{\sigma^{\prime} \sigma}(Q) \equiv \\
& * \Gamma^{000 *} \Delta^{l *} \Gamma^{000 *} \Delta^{l}+{ }^{*} \Gamma^{00 i}\left(-g^{i j}+k^{i} k^{j}\right) \\
& \times{ }^{*} \Delta^{t *} \Gamma^{j 00 *} \Delta^{l}+{ }^{*} \Gamma^{j 00 *} \Delta^{l *} \Gamma^{00 i}\left(-g^{i j}+q^{i} q^{j}\right)^{*} \Delta^{t} \\
& +{ }^{*} \Gamma^{n 0 i}\left(-g^{i j}+k^{i} k^{j}\right)^{*} \Delta^{t} \\
& \times{ }^{*} \Gamma^{j 0 m}\left(-g^{m n}+q^{m} q^{n}\right)^{*} \Delta^{t} .
\end{aligned}
$$

The effective gluon vertex functions are of the following form:

$$
{ }^{*} \Gamma^{(n)}=\Gamma^{(n)}+\delta \Gamma^{(n)}, n=3,4 .
$$

In this expression, the quantity $\Gamma^{3(4)}$ is the bare three (four)-gluon vertex, which is equal to zero when the number of external retarded indices is 0 or 2 , and the quantity $\delta \Gamma^{3(4)}$ is the corresponding HTL contribution. The expressions of $\delta \Gamma^{3(4)}$ are derived and grouped in two groups:

1. The number of $r$ 's occurring in the vertex is 1 or 3: Any vertex belonging to this group has the bare vertex and can be obtained via a direct analytic continuation of the already known HTL in the imaginary-time formalism, and is proportional to $g^{2} T^{2}$.

2. The number of $r$ 's occurring in the vertex is 0 or 2 : Any vertex belonging to this group does not have the bare term and is proportional to $g^{2} T^{3}$.

In the process of determining the hard-thermal-loop vertex functions, we have first to perform the angular integrals in The HTL 3-gluon and 4-gluon vertices. This could be done using the Feynman parameterization. As an illustration, let us discuss how to manipulate the HTL contribution of same terms in the 3-gluon vertices. We choose the following term:

$$
\begin{aligned}
\delta & \Gamma_{\mathrm{raa}}^{\mathrm{000}}(P,-K,-Q)= \\
& -m_{g}^{2} \int \frac{\mathrm{d} \Omega_{S}}{4 \Pi}\left[-\frac{p_{0}}{(Q S-\mathrm{i} \varepsilon)(P S-\mathrm{i} \varepsilon)}\right.
\end{aligned}
$$

$$
\begin{aligned}
& \left.+\frac{k_{0}}{(Q S-\mathrm{i} \varepsilon)(K S-\mathrm{i} \varepsilon)}\right]= \\
& m_{g}^{2} \int_{0}^{1} \mathrm{~d} y \int \frac{\mathrm{d} \Omega_{S}}{4 \Pi}\left[\frac{p_{0}}{\left(R_{1}(y) S-\mathrm{i} \varepsilon\right)^{2}}\right. \\
& \left.-\frac{k_{0}}{\left(R_{2}(y) S-\mathrm{i} \varepsilon\right)^{2}}\right]
\end{aligned}
$$

where $R_{1}(y)=((Q-P) y+P), R_{2}(y)=((Q-K) y+$ $K), S \equiv\left(1, s^{i}\right)$ and $\Omega_{S} \equiv(\phi, \theta)$ is the solid angle of $\hat{s}\left(R S=r_{0}-\hat{r} \cdot \hat{s}\right)$. In order to perform the solid-angle integral, we measure the solid angle with respect to $\hat{r}_{1}$ in the first integral and with respect to $\hat{r}_{2}$ in the second. We get:

$$
\begin{aligned}
& \delta \Gamma_{\text {raa }}^{000}(P,-K,-Q)=m_{g}^{2} \int_{0}^{1} \mathrm{~d} y \\
& \quad \times\left[\frac{p_{0}}{\left(r_{01}-\mathrm{i} \varepsilon\right)^{2}-r_{1}^{2}}\right. \\
& \left.-\frac{k_{0}}{\left(r_{02}-\mathrm{i} \varepsilon\right)^{2}-r_{2}^{2}}\right]
\end{aligned}
$$

and we have terms of the following form:

$$
\begin{aligned}
& \delta \Gamma_{\text {raa }}^{0 i 0}(P,-K,-Q)=-m_{g}^{2} \int_{0}^{1} \mathrm{~d} y \int \frac{\mathrm{d} \Omega_{S}}{4 \Pi} \hat{s}^{i} \\
& \times\left(-\frac{k_{0}}{\left(R_{1}(y) S-\mathrm{i} \varepsilon\right)^{2}}+\frac{k_{2}}{\left(R_{2}(y) S-\mathrm{i} \varepsilon\right)^{2}}\right)= \\
& m_{g}^{2} \int_{0}^{1} \mathrm{~d} y\left(p _ { 0 } \left\{\frac{1}{4 r_{1}^{2}} \ln \left(\frac{\left(r_{01}-r_{1}\right)^{2}+\varepsilon^{2}}{\left(r_{01}+r_{1}\right)^{2}+\varepsilon^{2}}\right)\right.\right. \\
& +\frac{\mathrm{i}}{2 r_{1}^{2}}\left[\arctan \left(\frac{r_{01}-r_{1}}{\varepsilon}\right)-\arctan \left(\frac{r_{01}+r_{1}}{\varepsilon}\right)\right] \\
& \left.+\frac{r_{01}-\mathrm{i} \varepsilon}{r_{1}\left(r_{01}-\mathrm{i} \varepsilon\right)^{2}-r_{1}^{3}}\right\} \hat{r}_{1} \\
& -k_{0}\left\{\frac{1}{4 r_{2}^{2}} \ln \left(\frac{\left(r_{02}-r_{2}\right)^{2}+\varepsilon^{2}}{\left(r_{02}+r_{2}\right)^{2}+\varepsilon^{2}}\right)\right. \\
& +\frac{\mathrm{i}}{2 r_{2}^{2}}\left[\arctan \left(\frac{r_{02}-r_{2}}{\varepsilon}\right)-\arctan \left(\frac{r_{02}+r_{2}}{\varepsilon}\right)\right] \\
& \left.\left.+\frac{r_{02}-\mathrm{i} \varepsilon}{r_{2}\left(r_{02}-\mathrm{i} \varepsilon\right)^{2}-r_{2}^{3}}\right\} \hat{r}_{2}\right) .
\end{aligned}
$$

The other solid-angle integrals in the HTL vertices are calculated separately in a similar manner. Putting things together and replacing the effective propagators by their expressions, we get the analytic expression of the effective self-energy of longitudinal gluons. The resulting integrals themselves will have to be performed numerically.

\section{Conclusions}

In this communication, we have reported on how to calculate the next-to-leading order energy for slow-moving 
longitudinal gluons in the RTF of HTL perturbation theory of massless QCD at high temperature. The effective vertex functions in RTF are derived and the angular integral in the HTL vertex functions is done analytically. A compact analytic expression for the complete next-toleading contribution to the retarded longitudinal gluon self-energy is also given. The next phase in this calculation is to numerically evaluate the resulting integrals. This work is in progress.

\section{References}

[1] E. Braaten, R.D. Pisarski, Nucl. Phys. B 337, 569 (1990).

[2] M. Le Bellac, Quantum statistical mechanics, Thermal Field Theory, Cambridge University Press, 1996.
[3] E. Braaten, R.D. Pisarski, Phys. Rev. D 42, 2156 (1990).

[4] A. Abada, O. Azi, K. Benchallal, Phys. Lett. B 425, 158 (1998).

[5] A. Abada, K. Bouakaz, O. Azi, Phys. Scr. 74, 77 (2006).

6] A. Abada, K. Bouakaz, N. Daira-Aifa, Eur. Phys. J. C. 18, 765 (2001).

[7] M.E. Carrington, Phys. Rev. D 75, 045019 (2007).

[8] M.E. Carrington, A. Gynther, D. Pickering, Phys. Rev. D 78, 045018 (2008).

[9] Y. Fueki, H. Nakkagawa, H. Yokota, K. Yoshida, Prog. Theor. Phys. 107, 759 (2002). 